\title{
Precisely positioning pallets in multi-station assembly systems
}

\author{
R. Ryan Vallance ${ }^{\mathrm{a}, *}$, Chris Morgan ${ }^{\mathrm{b}, 1}$, Alexander H. Slocum ${ }^{\mathrm{c}, 2}$ \\ ${ }^{a}$ Department of Mechanical and Aerospace Engineering, The George Washington University, \\ 738 Academic Center Bldg., 801 22nd St., N.W., Washington, DC 20052, USA \\ ${ }^{\mathrm{b}}$ Department of Mechanical Engineering, University of Kentucky, 015 Ralph G. Anderson Building, Lexington, KY 40506, USA \\ c Department of Mechanical Engineering, Massachusetts Institute of Technology, RM 3-445 77 Massachusetts Ave., Cambridge, MA 02139, USA
}

Received 28 August 2002; accepted 14 November 2002

\begin{abstract}
In multi-station assembly systems, common for mass-customization manufacturing strategies, the product being assembled is held in a fixture attached to a pallet, and the pallet is conveyed between workstations. In high-precision assembly systems, variation in the position of the pallet is one of the largest sources of variation within the error budget, reducing quality and yields. Conventional approaches to locating pallets use pins and bushings, and a method for predicting their repeatability is presented. This paper also presents an exact constraint approach using a split-groove kinematic coupling, which reduces variation in pallet location by an order of magnitude.
\end{abstract}

(C) 2003 Elsevier Inc. All rights reserved.

Keywords: Kinematic couplings; Split-groove coupling; Pallets; Flexible assembly systems; Assembly automation

\section{Introduction}

Nearly all products require that manufactured parts be assembled together into a final product. The assembly process is conducted manually and/or automatically using assembly machines. The term "assembly automation", popularized around World War II, refers to the mechanized feeding, placement, and fastening of manufactured components into a complete product assembly. Since tooling and equipment is often dedicated to a particular product, automated assembly is generally reserved for mass-produced components, where the expense of custom tooling and equipment can be distributed over many units. During the 1970s and 1980s, much engineering and research effort concentrated on exchanging hard automation for flexible automation by incorporating re-programmable equipment such as robots and computer controlled machinery. Flexible automation prolonged the life of capital equipment, and consequently, automated assembly became economical for lower-volume production. More recently, reduced time-to-market and shortened product life cycles provided further incentive for flexible automation using computer-controlled equipment. For these reasons,

\footnotetext{
* Corresponding author. Tel.: +1-202-994-9830; fax: +1-202-994-0238.

E-mail addresses: vallance@gwu.edu (R.R. Vallance), cjmorg1@engr.uky.edu (C. Morgan), slocum@mit.edu (A.H. Slocum).

${ }^{1}$ Tel.: +1-859-257-6336x80676; fax: +1-859-257-3304.

${ }^{2}$ Tel.: +1-617-253-0012; fax: +1-617-258-6427.
}

automated assembly machinery is now commonly used for many products.

To achieve economical production, assembly tasks are frequently executed simultaneously on several product units using multiple machines. This generally requires that the machines or workstations be organized into an assembly system, and the products are then transported between machines or manual workstations. There are three principal categories of assembly systems: synchronous, non-synchronous, or continuous assembly systems. Synchronous systems cyclically index the products to individual machines simultaneously with fixed frequency. In non-synchronous systems, the assembly machines operate independently, and the index time depends upon the task time at each machine. Non-synchronous systems therefore employ buffers between the machines or stations to accommodate different task times at each station. In continuous systems, the product remains in constant motion, and the tasks are performed while the product travels.

All three types of assembly systems require that the product be transported through the system. In synchronous and non-synchronous systems, the product units are stationary at each assembly machine or workstation. Therefore, the product unit must be statically positioned with respect to each assembly machine. A common approach is to hold each product unit in a fixture attached to an assembly pallet as illustrated in Fig. 1a. Then the pallets can be moved synchronously or non-synchronously throughout the assembly system using a pallet transportation system. Multi-station assembly systems 


\begin{tabular}{|c|c|}
\hline \multicolumn{2}{|c|}{ Nomenclature } \\
\hline$a_{i}$ & radius of contact area at contact point $i$ \\
\hline$c$ & clearance between pin and hole \\
\hline$d_{\mathrm{h}}$ & distance between holes \\
\hline$d_{\mathrm{p}}$ & distance between pins \\
\hline$D_{\mathrm{h}}$ & diameter of holes \\
\hline$D_{\mathrm{p}}$ & diameter of pins \\
\hline$D_{\mathrm{s}_{i}}$ & diameter of sphere at contact point $i$ \\
\hline$E_{\mathrm{e}}$ & equivalent modulus of elasticity \\
\hline$E_{\mathrm{f}}$ & modulus of elasticity for flat surface \\
\hline$E_{\mathrm{s}}$ & modulus of elasticity for spherical surface \\
\hline$[K]$ & kinematic coupling's system matrix \\
\hline$l_{\mathrm{CD}}$ & length of line $\mathrm{CD}$ \\
\hline$l_{\mathrm{CE}}$ & length of line $\mathrm{CE}$ \\
\hline$l_{\mathrm{DE}}$ & length of line $\mathrm{DE}$ \\
\hline$\vec{L}_{\mathrm{A}}$ & vector $(6 \times 1)$ of applied forces and moments \\
\hline$\vec{L}_{\mathrm{D}}$ & $\begin{array}{l}\text { vector }(6 \times 1) \text { of disturbance forces and } \\
\text { moments }\end{array}$ \\
\hline$\vec{L}_{\mathrm{P}}$ & vector $(6 \times 1)$ of preload forces and moments \\
\hline$\vec{L} \mathrm{~W}$ & vector $(6 \times 1)$ of weight forces and moments \\
\hline$\vec{n}_{i}$ & unit normal vector at contact point $i$ \\
\hline$\vec{P}_{\mathrm{B}_{j}}$ & position vector locating ball $j$ \\
\hline$P_{\mathrm{C}_{i}}$ & position vector locating contact point $i$ \\
\hline$\vec{P}_{\mathrm{E}}$ & $\begin{array}{l}\text { position vector locating an operating point } \\
\text { after error motion }\end{array}$ \\
\hline$P_{\mathrm{o}}$ & $\begin{array}{l}\text { position vector locating an operating point } \\
\text { before error motion }\end{array}$ \\
\hline$\vec{R}_{\mathrm{C}}$ & vector $(6 \times 1)$ of contact reaction forces \\
\hline$\left[T_{\mathrm{E}}\right]$ & $\begin{array}{l}\text { homogenous transformation matrix } \\
\text { representing error motion }\end{array}$ \\
\hline$x$ & coordinate of a point in the $x$ direction \\
\hline$x_{\mathrm{D}}$ & coordinate of point $\mathrm{D}$ in the $x$ direction \\
\hline$\hat{x}_{\mathrm{D}}$ & $\begin{array}{l}\text { approximate coordinate of point } \mathrm{D} \text { in } x \\
\text { direction }\end{array}$ \\
\hline$x_{\mathrm{E}}$ & coordinate of point $\mathrm{E}$ in $x$ direction \\
\hline$\hat{x}_{\mathrm{E}}$ & $\begin{array}{l}\text { approximate coordinate of point } \mathrm{E} \text { in } x \\
\text { direction }\end{array}$ \\
\hline$y$ & coordinate of a point in the $y$ direction \\
\hline$y_{\mathrm{D}}$ & coordinate of point $\mathrm{D}$ in the $y$ direction \\
\hline$\hat{y}_{\mathrm{D}}$ & $\begin{array}{l}\text { approximate coordinate of point } \mathrm{D} \text { in } y \\
\text { direction }\end{array}$ \\
\hline$y_{\mathrm{E}}$ & coordinate of point $\mathrm{E}$ in $y$ direction \\
\hline & $\begin{array}{l}\text { approximate coordinate of point } \mathrm{E} \text { in } y \\
\text { direction }\end{array}$ \\
\hline \multicolumn{2}{|c|}{ Greek letters } \\
\hline$\vec{\delta}$ & translation error \\
\hline$\delta_{\mathrm{c}}$ & $\begin{array}{l}\text { vector }(6 \times 1) \text { of elastic deformation at } \\
\text { contact points }\end{array}$ \\
\hline$\delta_{\mathrm{c}_{i}}$ & elastic deformation at contact point $i$ \\
\hline$\delta \mathrm{E}$ & $\begin{array}{l}\text { vector }(6 \times 1) \text { of error motion translations } \\
\text { and rotations }\end{array}$ \\
\hline
\end{tabular}

\begin{tabular}{|c|c|}
\hline$\delta_{x}$ & $\begin{array}{l}\text { component of translation error in the } x \\
\text { direction }\end{array}$ \\
\hline$\delta_{y}$ & $\begin{array}{l}\text { component of translation error in the } y \\
\text { direction }\end{array}$ \\
\hline$\theta$ & range of rotational error \\
\hline$\theta_{\max }$ & maximum range of rotational error \\
\hline$v_{\mathrm{f}}$ & Poisson's ratio for flat surface \\
\hline$v_{\mathrm{s}}$ & Poisson's ratio for spherical surface \\
\hline$\rho$ & estimate of repeatability \\
\hline$\sigma_{\max _{i}}$ & maximum tensile stress at contact point $i$ \\
\hline$\tau_{\max _{i}}$ & maximum shear stress at contact point $i$ \\
\hline
\end{tabular}

often use conveyor belts as illustrated in Fig. 1b, and they generally operate non-synchronously.

This paper presents designs and techniques for precisely positioning assembly pallets at individual machines within assembly systems. Two separate approaches, pins-in-holes and exact constraint, are compared on the basis of stiffness and positional repeatability. It is shown that the repeatability of the exact constraint approach is much better than with the pins-in-holes approach. Although the static stiffness of the pins-in-holes approach can be greater, the stiffness of exact constraint is usually sufficient for most assembly tasks. Therefore, in precision assembly systems, the advantage of positional repeatability using exact constraint often outweighs additional improvement in static stiffness.

\section{Positioning with pins in holes}

A common technique for positioning one body with respect to another is to mate a set of pins (fixed in one body) inside a set of holes (in the second body). This pins-in-holes (PIH) technique is non-deterministic since contact between the two bodies may or may not occur. If contact occurs, it is difficult to anticipate the locations of contact, quantity, or sizes of any contact regions. This uncertainty arises from manufacturing errors in:

(1) distances between the pins or holes,

(2) diameters of the pins or holes,

(3) straightness of the pins or holes,

(4) parallelism of the pins or holes, and

(5) cylindricity of the pins or holes.

Since PIH approaches are non-deterministic, their performance (repeatability and stiffness) is difficult to analyze or predict a priori, especially if more than two pins and two holes are used. The relation between design parameters (e.g. diameter of pins, clearance between pins and holes, distances between pins/holes) and performance cannot be expressed analytically for PIH configurations. As a result, it is often necessary to experimentally measure the repeatability and stiffness with actual manufacturing errors. For these reasons, we are limited to estimating planar repeatability for PIH de- 


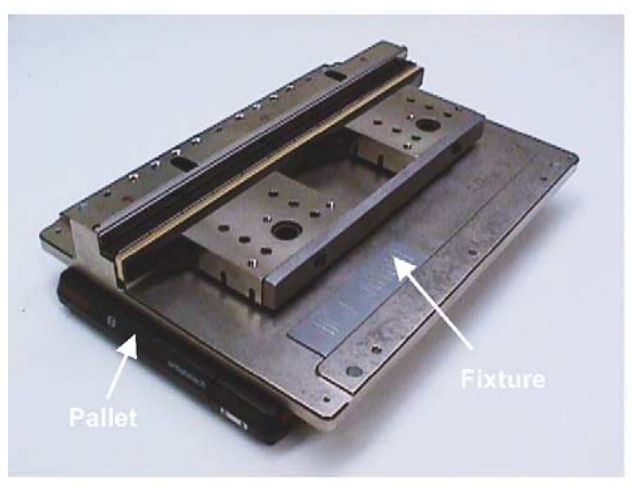

(a)

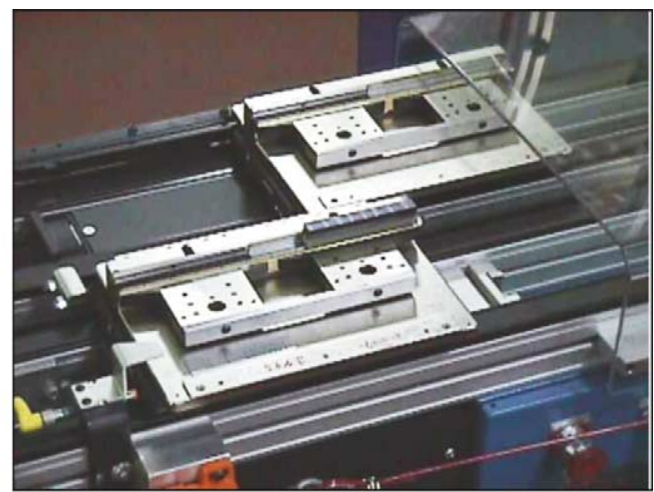

(b)

Fig. 1. (a) Pallet and fixture for holding electrical connector during assembly operations and (b) flexible assembly system with conveyors for non-synchronously transporting pallets.

signs employing only two pins and two holes (Section 2.2) and a qualitative discussion of static stiffness (Section 2.3).

\subsection{Design of pins-in-holes positioning technique}

A PIH positioning system may use any quantity of pins and holes, and as the quantity of pins and holes increases, the precision of the positioning improves and the stiffness increases. However, systems with more than two holes and two pins require tighter tolerances to prevent binding while mating the two objects. If the clearance between the pins and holes is insufficient to accommodate manufacturing errors then binding occurs, and large forces are necessary to elastically deform the pins and holes. Design precautions, which degrade the precision and stiffness, are necessary to reduce the chance of binding due to manufacturing errors.

The most obvious and common design precaution is to increase the clearance between the outer diameter (OD) of the pins and the inner diameter (ID) of the holes by manufacturing the holes slightly larger than the pins. This precaution accommodates many types of manufacturing errors, but dramatically reduces repeatability and stiffness of the PIH design. Another precaution reduces the sensitivity to manufacturing errors in the roundness, straightness, and parallelism by minimizing the length of engagement between the pin and hole. This is frequently accomplished using pins with large rounds or bullet noses that lead into shorter cylindrical regions, such as those shown in Fig. 2. A third design pre-

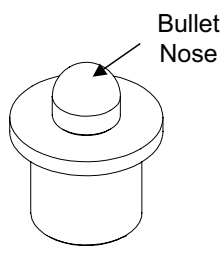

(a)

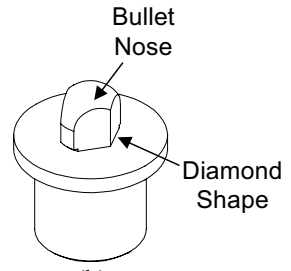

(b)

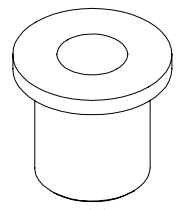

(c)
Fig. 2. Commercially available positioning pins and bushing (a) round pin with bullet nose, (b) diamond pin with bullet nose, and (c) bushing. caution uses one round pin (Fig. 2a) and one diamond shaped pin (Fig. 2b) to reduce the sensitivity to a difference in the distance between pins and the distance between holes. The diamond pin is manufactured with two sharp corners and two rounded corners. The rounded corners have approximately the radius of an equivalent round pin. The distance between the two sharp corners should be less than the diameter of an equivalent round pin, and the distance between the two rounded corners is approximately equal to the diameter of an equivalent round pin. The diamond pin is oriented during assembly so that one of the sharp corners points directly toward the round pin. This insures that the rounded corners point perpendicular to a line connecting the centers of the two pins.

Fig. 3 illustrates an industry standard pallet that uses the PIH positioning technique [1]. In this pallet system, two of the design precautions are applied. A round pin is used along with a diamond pin, and the tolerances are such that clearance is guaranteed between the pins and holes. The pallet is constructed of a steel or aluminum plate surrounded with a polyimide frame. Hard bushings with ground bores are pressed into the plate, and hard pins with ground surfaces are attached to the machine or assembly station (represented by plate in Fig. 3).

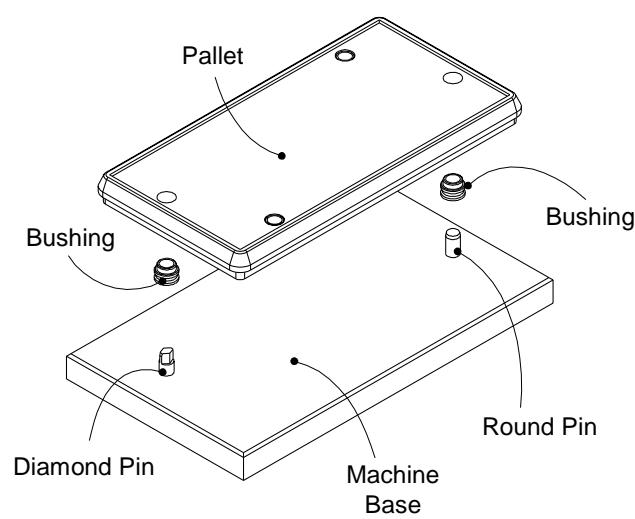

Fig. 3. Position method in commercial pallets using pins-in-holes approach (distance between pins equals $287.5 \mathrm{~mm}$ (11.321 in.)). 
Table 1

Dimensions and tolerance of pins and bushing holes for industry standard pallet

\begin{tabular}{|c|c|c|c|}
\hline Object & Geometry & Nominal diameter dimension & $\begin{array}{l}\text { Diameter tolerance specification } \\
\text { and tolerance range }\end{array}$ \\
\hline Round pin & & $12 \mathrm{~mm}$ (0.4724 in.) & $\begin{array}{l}\mathrm{e} 7 \\
-0.0305 \mathrm{~mm}(0.0012 \mathrm{in} .) \\
-0.0483 \mathrm{~mm}(0.0019 \mathrm{in} .)\end{array}$ \\
\hline Diamond pin & & $12 \mathrm{~mm}(0.4724 \mathrm{in})$. & $\begin{array}{l}\mathrm{e} 7 \\
-0.0305 \mathrm{~mm}(0.0012 \mathrm{in} .) \\
-0.0483 \mathrm{~mm}(0.0019 \mathrm{in} .)\end{array}$ \\
\hline Round bushing & & $12 \mathrm{~mm}(0.4724 \mathrm{in})$. & $\begin{array}{l}\mathrm{H} 7 \\
+0.0178 \mathrm{~mm}(0.0007 \mathrm{in} .) \\
-0.0000 \mathrm{~mm}(0.0000 \mathrm{in} .)\end{array}$ \\
\hline
\end{tabular}

The geometry, dimensions, and tolerance for the ground pins and bushings for the pallet system illustrated in Fig. 3 are summarized in Table 1. According to the tolerance specifications, the minimum pin diameter is $11.952 \mathrm{~mm}$ (0.4705 in.), and the maximum bushing bore diameter is $12.0178 \mathrm{~mm}$ (0.4731 in.). Hence, the worst-case diametric clearance is about $66 \mu \mathrm{m}$ (0.0026 in.). The manufacturer of the pallet system illustrated in Fig. 3 specifies that their pallets are repeatable to within $\pm 50 \mu \mathrm{m}$ ( \pm 0.002 in.) [1].

\subsection{Estimating precision of pins-in-holes positioning}

The precision, or two-dimensional repeatability, of a particular PIH design is difficult to predict a priori since the pallet's position is non-deterministic and depends upon manufacturing errors. However, an estimate of the worst-case limit on repeatability can be obtained as an envelope that bounds possible translation and rotational errors. This section presents a technique for determining that envelope for $\mathrm{PIH}$ designs that use a bullet-nose round pin, a bullet-nose diamond pin, and two bushings. The clearance is assumed sufficient to prevent binding. The diameter and geometric form of the pins and holes is assumed perfect (roundness and straightness), and contact is assumed to occur only around the circumference of the pins. The clearance between a pin and hole, $c$, is defined in Eq. (1) to be the difference between the nominal diameter of the pins, $D_{\mathrm{p}}$, and nominal diameter of the holes, $D_{\mathrm{h}}$ :

$c=D_{\mathrm{h}}-D_{\mathrm{p}}$

Fig. 4 illustrates the errors between two rigid bodies connected with a PIH design using two pins and two holes. The distance between the two pins is $d_{\mathrm{p}}$, and the distance between the two holes is $d_{\mathrm{h}}$. The clearance between the pins and holes is exaggerated for illustrative purpose. Since PIH is a planar positioning technique, we are only concerned with errors in three degrees of freedom: translation in the $x$ direction, translation in the $y$ direction, and a rotation angle. Two crosshatched circles represent the pins in the stationary object (machine base or workstation). The errors are measured in a reference coordinate system with origin, $\mathrm{O}$, located at the center of Pin 1 with $x$-axis pointing towards the center of Pin 2.

The coupled body (pallet) is shown in two distinct orientations. The two orientations are the extreme cases of rotational error, limited when Hole 2 contacts Pin 2. In the first orientation (solid lines with crosshatching), Pin 2 contacts Hole 2 at Point $\mathrm{A}$, and in the second orientation (dashed lines without crosshatching) Pin 2 contacts Hole 2 at Point B. Both orientations are illustrated with the same translation error, $\vec{\delta}$, that has components $\delta_{x}$ and $\delta_{y}$ in the $x$ and $y$ directions of the reference coordinate system located at the center of Pin 1.

The potential range of rotational error, $\theta$, depends upon the direction and magnitude of the translation error, $\vec{\delta}$. For instance, a translation error solely in the $x$ direction with magnitude equal to the clearance will not permit any rotational error, but a translation error solely in the $y$ direction with magnitude equal to the clearance permits rotational error. Therefore, an expression for the range of permissible rotational error as a function of translation errors is needed to evaluate the potential repeatability errors of a PIH design.

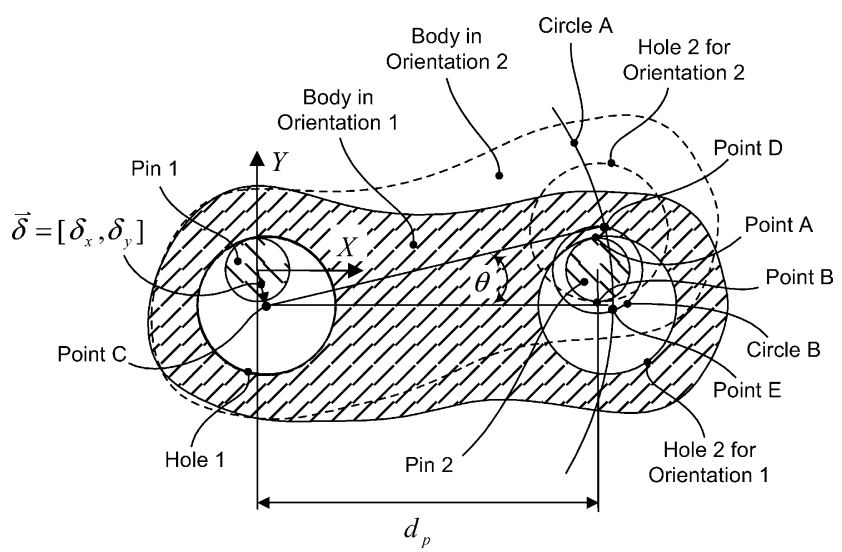

Fig. 4. Errors in pin-in-hole positioning technique. 
The centers of Hole 2 in the two extreme orientations lie at the intersections of two theoretical circles, labeled as Circle $\mathrm{A}$ and Circle B in Fig. 4. The center of Circle $\mathrm{A}$ is at the center of Hole 1 (Point $\mathrm{C}$ ), and its radius is equal to the distance between the two holes, $d_{\mathrm{h}}$. Circle A represents the locus of points traced by the center of Hole 2 when a rotation occurs about Point C. Points that lie on Circle A satisfy Eq. (2):

$\left(x-\delta_{x}\right)^{2}+\left(y-\delta_{y}\right)^{2}=d_{\mathrm{h}}^{2}$

The center of Circle B is at the center of Pin 2, and its radius equals the difference between the radii of the hole and pin. The radius of Circle $\mathrm{B}$ equals the maximum distance between the center of Pin 2 and Hole 2 that can ever occur. Points that lie on Circle B satisfy Eq. (3), which is written in terms of the diametric clearance, $c$ :

$\left(x-d_{\mathrm{p}}\right)^{2}+y^{2}=\frac{c^{2}}{4}$

In the two extreme orientations, the center of Hole 2 could be located at either Point $\mathrm{D}$ or E. The coordinates of Points $\mathrm{D}$ and $\mathrm{E}$ in the reference coordinate system can be determined by solving Eqs. (2) and (3) as a system of simultaneous non-linear equations. The coordinates of Points $\mathrm{D}$ and $\mathrm{E}$ are designated as $\left(x_{\mathrm{D}}, y_{\mathrm{D}}\right)$ and $\left(x_{\mathrm{E}}, y_{\mathrm{E}}\right)$, respectively, and can be readily calculated using iterative techniques. Once the coordinates Points D and E are determined, the lengths of edges along triangle $\mathrm{CDE}$ can be calculated. The lengths of the triangles edges $\left(l_{\mathrm{DE}}, l_{\mathrm{CD}}\right.$, and $\left.l_{\mathrm{CE}}\right)$ are given by Eqs. (4)-(6):

$l_{\mathrm{DE}}=\left[\left(x_{\mathrm{E}}-x_{\mathrm{D}}\right)^{2}+\left(y_{\mathrm{E}}-y_{\mathrm{D}}\right)^{2}\right]^{1 / 2}$

$l_{\mathrm{CD}}=\left[\left(x_{\mathrm{D}}-\delta_{x}\right)^{2}+\left(y_{\mathrm{D}}-\delta_{y}\right)^{2}\right]^{1 / 2}$

$l_{\mathrm{CE}}=\left[\left(x_{\mathrm{E}}-\delta_{x}\right)^{2}+\left(y_{\mathrm{E}}-\delta_{y}\right)^{2}\right]^{1 / 2}$

The potential range of rotational error, $\theta$, equals the angle between the edges CD and CE. Since the three edges' lengths are known, the angle $\theta$ can be calculated using the Law of Cosines as shown in Eq. (7):

$\theta=f\left(\delta_{x}, \delta_{y}\right)=\cos ^{-1}\left\{\frac{l_{\mathrm{CD}}^{2}+l_{\mathrm{CE}}^{2}-l_{\mathrm{DE}}^{2}}{2 l_{\mathrm{CD}} l_{\mathrm{CE}}}\right\}$

Although Eqs. (2)-(7) yield a general solution, the analysis for most PIH systems, including pallet positioning, can be approximated with a simpler closed-form solution that does not require iterative methods. The approximate solution is derived with a few reasonable assumptions. Small clearances are assumed so that $D_{\mathrm{h}}$ and $D_{\mathrm{p}}$ are approximately equal, and the distances between the two pins and two holes are assumed to be equal (so that $d_{\mathrm{p}}$ equals $d_{\mathrm{h}}$ ). The coordinates for Points $\mathrm{D}$ and $\mathrm{E}$ approximated with these assumptions is distinguished using $\wedge$ above the variables, $\hat{x}_{\mathrm{D}}, \hat{y}_{\mathrm{D}}$ and $\hat{x}_{\mathrm{E}}, \hat{y}_{\mathrm{E}}$. The approximate coordinates in the $x$-direction, $\hat{x}_{\mathrm{D}}$ and $\hat{x}_{\mathrm{E}}$, are equal; their value is simply the sum of the translation error in the $x$ direction and the distance between the pins as given in Eq. (8). The approximate $y$ coordinates, $\hat{y}_{\mathrm{D}}$ and $\hat{y}_{\mathrm{E}}$, for the centers of Hole 2 in both orientations are given by Eqs. (9) and (10), respectively:

$\hat{x}_{\mathrm{D}} \approx \hat{x}_{\mathrm{E}} \approx \delta_{x}+d_{\mathrm{p}}$

$\hat{y}_{\mathrm{D}} \approx\left[\frac{c^{2}}{4}-\delta_{x}^{2}\right]^{1 / 2}$

$\hat{y}_{\mathrm{E}} \approx-\left[\frac{c^{2}}{4}-\delta_{x}^{2}\right]^{1 / 2}$

For most applications, the distance between the pins is much greater than the clearance $\left(d_{\mathrm{p}} \gg c\right)$, and therefore, $l_{\mathrm{CD}}$ and $l_{\mathrm{CE}}$ are approximately equal the distance between the pins, $d_{\mathrm{p}}$. Substituting the approximate coordinates from Eqs. (8) to $(10)$ and $d_{\mathrm{p}}$ for $l_{\mathrm{CD}}$ and $l_{\mathrm{CE}}$ into Eq. (7) yields a closed-form estimate for the potential range of orientation error, $\hat{\theta}$. Note that the approximate range of angular error does not depend upon $\delta_{y}$ :

$\hat{\theta}=f\left(\delta_{x}\right)=\cos ^{-1}\left(1-\frac{c^{2}}{2 d_{\mathrm{p}}^{2}}+\frac{2 \delta_{x}^{2}}{d_{\mathrm{p}}^{2}}\right)$

From Eq. (11), it is evident that the maximum rotational error, $\hat{\theta}_{\max }$, occurs when the translation error is zero $\left(\delta_{x}=0\right)$. Accordingly, the approximate maximum rotation error is given in Eq. (12). This result matches engineering intuition in that reducing the clearance and increasing the distance between pins reduces rotational errors:

$\hat{\theta}_{\max }=\cos ^{-1}\left[1-\frac{c^{2}}{2 d_{\mathrm{p}}^{2}}\right]$

Finally, an envelope bounding the repeatability errors for a particular PIH design can be specified. Due to the circular perimeter of holes and pins, the translations in both the $x$ and $y$ directions are limited within a circle of radius $c / 2$; hence, the translation errors are bounded by $-c / 2$ and $c / 2$. The rotational errors are bounded within $-\hat{\theta}_{\max } / 2$ and $\hat{\theta}_{\max } / 2$.

A visual interpretation of these limits to repeatability errors is obtained by graphically plotting $\theta_{\max }$ or $\hat{\theta}_{\max }$ as a function of $\delta_{x}$ and $\delta_{y}$. The translation errors in the $x$ and $y$ directions are plotted along the $x$ and $y$ axes, and the range of rotational error is plotted along the $z$-axis. Fig. 5 shows a graph of the repeatability limits for the examplary PIH pallet system shown in Fig. 3 with $d_{\mathrm{p}}=287.5$ and $c=0.0661 \mathrm{~mm}$. The range of rotational errors shown in Fig. 5 was determined using the approximate solution method. For this particular $\mathrm{PIH}$ pallet system, the maximum range of rotational error is calculated to be around $2.3 \times 10^{-4}$ radians. This rotational error is amplified over the length of the pallet into a translation error of around $0.07 \mathrm{~mm}$. For this analysis, the difference between the estimated solution and the solution obtained by solving the simultaneous equations is only $-4.2 \times 10^{-7} \%$, and thus, the approximate solution is adequate. 


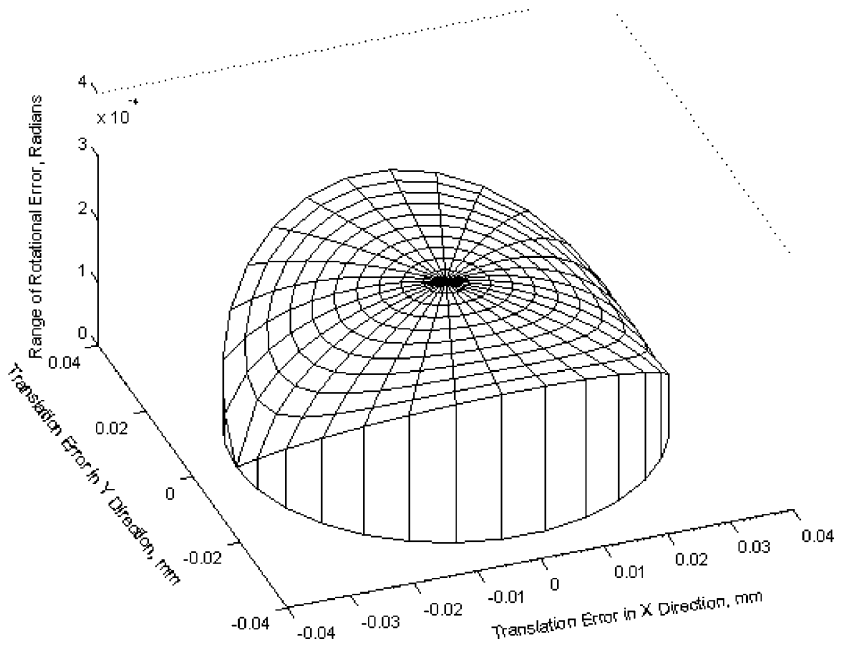

Fig. 5. Limits on planar repeatability errors in pallet system using pins-in-holes positioning.

\subsection{Stiffness of pins-in-holes positioning}

All assembly pallets are subjected to disturbance forces that occur during assembly operations. At each workstation, these forces have particular magnitudes, act in particular directions, and are applied at particular positions on the pallet, fixture, or workpiece. Disturbance forces produce elastic deformation in the pallet, fixture, workpiece, and at the contact regions between the pallet and the workstation. The contact stiffness due to the pallet's positioning technique affects the amount of deflection and errors that result from disturbance forces. In precision assembly systems, the fixture and pallet are stiff to minimize errors due to elastic deformation. Hence, elastic errors at the contact points are generally not negligible in the error budget and should be considered when evaluating a pallet positioning technique.

For PIH designs, the stiffness of the contact regions depends upon the clearances between the pins and holes, the size and quantity of any contact areas, amount of friction between the coupled bodies, and the degree of loading. Because of manufacturing variation in pallets, pins, and bushings, these parameters cannot be known deterministically, and therefore, the static stiffness of a PIH design cannot be predicted a priori. Therefore, the relationship between PIH design parameters and static stiffness is discussed qualitatively.

Disturbance forces applied in the $z$ direction (parallel to the axis of the pins) are typically transferred directly from the pallet to the workstation without transmitting much of the force through contact areas between the pins and holes. The contact areas in the $z$ direction are often large flat surfaces on the bottom of the pallet and large flat surfaces on the workstations. The stiffness in the $z$ direction depends, however, upon the geometry of the pallet and the elastic modulus of the materials. In the commercial pallet system, a polyimide frame contacts a metal plate in the workstation. With suitable construction and materials, substantial static stiffness in the $z$ direction is obtainable, and reasonable estimates of stiffness

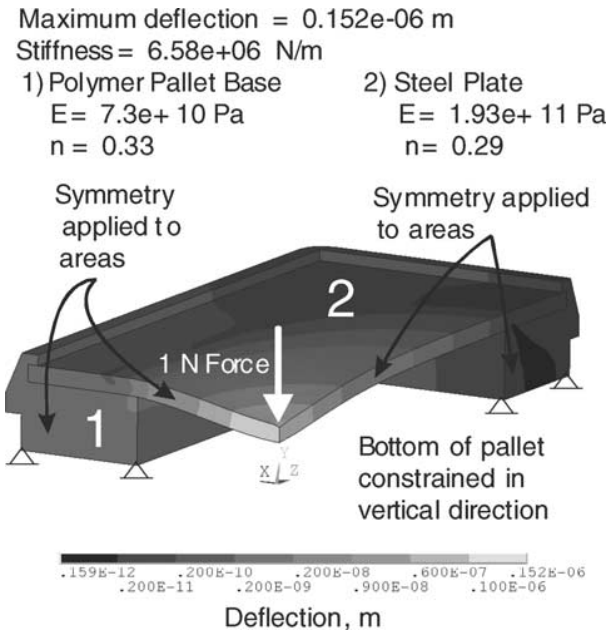

Fig. 6. Static stiffness of common commercial pallet using finite element analysis.

in the $z$ direction can be determined using elasticity models or finite element analyses (FEA). ${ }^{3}$ Fig. 6 shows the results of an FE analysis of the commercial pallet subjected to a force of $1 \mathrm{~N}$ in the center of the pallet. The static deflection is about $1.52 \times 10^{-7} \mathrm{~m}$, providing static stiffness of about $6.58 \times 10^{6} \mathrm{~N} / \mathrm{m}$.

Typically, the pins are used only for positioning, and so clamping forces are often applied to achieve lateral stiffness. With strong clamping forces, the stiffness for a particular PIH design can be quite large. However, if the pins are not initially in contact with the holes, then sliding (translation and/or rotation) might occur until the pins contact the holes, and the range of possible sliding errors can be estimated using the model described in Section 2.2. If clamping forces are insufficient and sliding occurs, then disturbance forces applied to the pallet in the tangential direction are transmitted from the pallet to the workstation through conforming contact between the pins and holes. When contact exists between the pin and holes, the stiffness depends upon the size of the contact area between the pins and the holes. With tight clearances (slip-fits and/or press-fits) and large engagement lengths, the stiffness is typically sufficient for pallets. However, this greatly compromises the ease of placing the pallet on the workstation and separating the pallet from the workstation. Thus most PIH pallet systems typically maintain clearance between the pins and holes and rely on clamping for lateral stiffness.

\section{Positioning with exact constraint devices}

An alternative approach for positioning pallets uses exact constraint devices between the pallets and workstations

\footnotetext{
${ }^{3}$ If the stiffness of the workstation is great compared to the stiffness of the pallet, then a reasonable FEA model can be obtained using zero-displacement boundary conditions on the pallet's bottom surface. If the stiffness of the workstation's structure is comparable or less stiff than the pallet, then the workstation structure should also be included in the FEA model.
} 


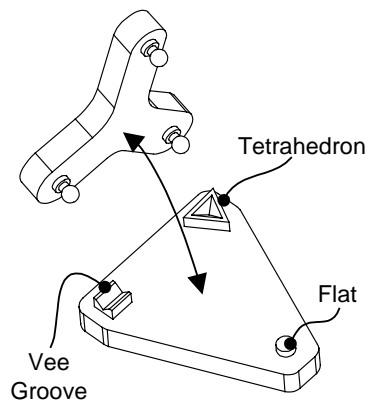

(a)

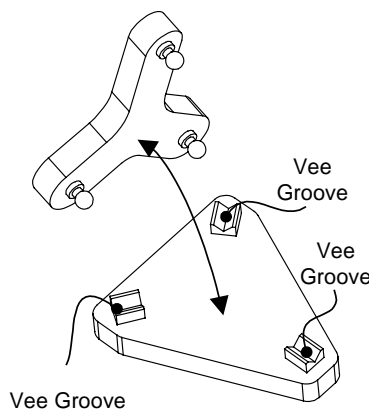

(b)

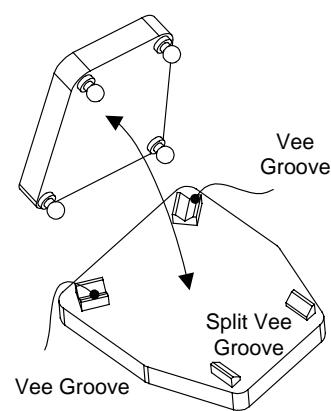

(c)

Fig. 7. Configurations of kinematic couplings. (a) Kelvin coupling, (b) three-groove coupling, and (c) the new split-groove coupling.

[2]. The term "exact constraint" refers to the fact that the pallet is neither over constrained nor under constrained [3]; sufficient constraints are applied to restrict exactly six degrees of freedom. Kinematic couplings are one form of exact constraint devices. Designers of instrumentation and optical systems have used kinematic couplings for many years [4-8], but their application in manufacturing processes and machines is more recent $[9,10]$. In pallet positioning, we consider the two bodies to be the machine base and the pallet. The challenge is to provide static stiffness and prevent tipping of the pallet when subjected to a variety of disturbance forces.

Kinematic couplings connect two bodies through contact at six points, exactly enough for static equilibrium. Six independent contact points ensures that the coupled body (pallet) is statically determinant but not over-constrained. Kinematic couplings come in a few alternative configurations that differ according to the relative locations of the six contact points, the geometry of the contacting surfaces, their preload mechanism, and the contacting materials. The two most common configurations are the Kelvin coupling and the three-groove coupling [4]. The Kelvin coupling, illustrated in Fig. 7a, establishes six contact points by mating three balls on the first body with the surfaces of a tetrahedron, a vee-groove, and a flat on the second body. The three-groove coupling, illustrated in Fig. 7b, establishes six contact points by mating three balls on the first body with the surfaces of three vee-grooves on the second body. The split-groove coupling in Fig. 7c establishes six contact points by mating four balls with two vee-grooves and a split vee-groove in the second body. The split-groove coupling is amenable to supporting rectangular plates, where geometric constraints prevent using the configurations illustrated in Fig. 7a or b. The tipping resistance, after contact is established at all six points, can be greater for a split-groove configuration since it increases frictional moments. Hale [11] described a similar split-groove configuration for use in an optics assembly for the National Ignition Facility at the Lawrence Livermore National Labs.

Kinematic couplings gain two significant advantages by ensuring that the body is statically determinant. First, no elastic strain or deformation is induced in the coupled body due to forced congruence at additional contact points or surfaces. The second advantage is that a preferred relative position and orientation between the two bodies exists (due to minimizing potential energy). Unfortunately, since the contact between the bodies is limited to six points, the reactions at the contact points are distributed over very small areas; thus Hertzian stresses at the contact points are large when large disturbance forces are applied to the pallet (or in some cases the workstation). The elastic strain at the contact points that arises with the contact stress also limits the static stiffness between the coupled bodies. In addition, unless the preload is sufficiently high, loads applied to the pallet may cause the pallet to tip.

\subsection{Design of split-groove kinematic couplings}

Since many aspects of kinematic couplings can be analyzed, the design procedure enables a deterministic [12] approach. Several design and analysis aspects of kinematic couplings are described in precision engineering literature $[13,14]$, so a comprehensive presentation of the methods is not necessary here. Instead, a synopsis of the procedure is presented along with appropriate references to supplementary literature. Fig. 8 illustrates a procedure for designing kinematic couplings that consists of design and analysis phases. The first step in the design phase is selecting a configuration for the kinematic coupling. Although the most common configurations are the Kelvin coupling and the three-groove coupling, the split-groove coupling presented here is more suitable for integration with conveyor lines and rectangular pallets. In these applications, the area beneath the pallet must remain clear to avoid the conveying or indexing system.

The second design step is selecting the topography of the contacting surfaces. The topography considers the geometric shape of the contacting surfaces and whether the pallet contains the vee-grooves or balls. A simple topography is the case of a spherical surface contacting a flat surface on the assembly workstation. This topography is easy to analyze and produce since precise balls are readily available for tooling applications and smooth flat surfaces are easily manufactured. Depending upon the application, other topographies 


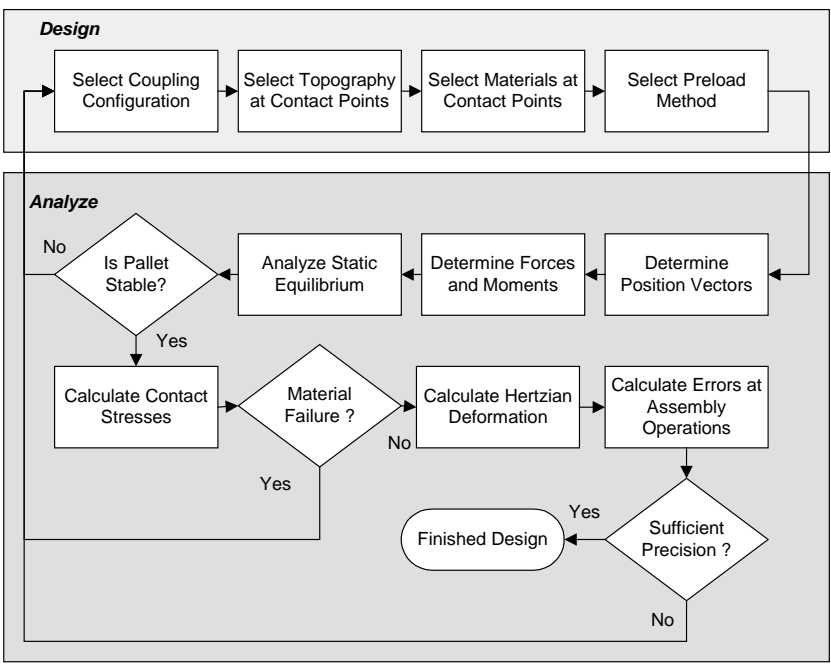

Fig. 8. Procedure for designing kinematic couplings for pallet systems.

such as those illustrated in Fig. 9 may be employed. For instance, employing conforming geometry such as a ball in a canoe can dramatically reduce contact stresses and increase contact stiffness. Van Doren described this technique to locate machinery modules for semiconductor manufacturing equipment [15].

The third design step is selecting appropriate materials for the contact points. The perfect material should have a high yield strength to withstand contact stresses, high hardness to minimize wear, and high toughness to prevent brittle fractures on the surface. For achieving greatest repeatability, the contacting materials should also have low friction (lubricant may be used in some cases). Schouten and Schellekens [16] showed that hysteresis due to friction can be dramatically reduced by cutting flexures into the grooves to give them tangential compliance while maintaining high stiffness in the direction of contact. Appropriate materials therefore include ceramics like tungsten carbide, silicon nitride, alumina, as well as hardened tool or stainless steels.

The final step is designing a method for preloading the pallet to the assembly workstation. Preload refers to a bias force that ensures contact between the pallet and the workstation regardless of disturbance forces. As described by Slocum

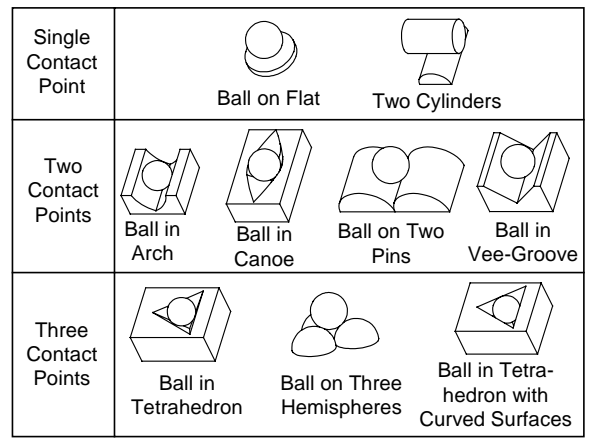

Fig. 9. Alternative contact topography for exact constraints.
[17], preload is essential in achieving precision, and two fundamental means are employed in mechanical systems: opposed geometry and applied external forces. Since opposed geometry (as used in recirculating ball bearings) requires the manufacture of extra high precision surfaces, applying external forces is often more economical. For example, permanent magnets were used in metrology equipment to apply external preload forces [18].

Blanding [3] describes a method to consider the placement and direction of preload forces. The method uses instantaneous centers of rotation and the geometric intersection of friction cones. The friction cones, with included angles equal to the friction angle, are constructed about the normal vectors at each contact point. To effectively nest a kinematic coupling, sliding must occur at the contact points. If preload forces pass through the geometric intersection of the friction cones, then sliding does not occur. The intersection of the friction cones is significantly smaller for a three-groove coupling than for a split-groove coupling. Therefore, care must be taken when specifying preload forces that will achieve reliable nesting in a split-groove configuration. When feasible, it is beneficial to place preload forces symmetrically near the contact points to minimize hysteresis due to pallet bending and friction.

\subsection{Analysis of pallets with split-groove kinematic couplings}

The analysis phase illustrated in Fig. 8 consists of six steps. First, a model of the kinematic coupling's geometry and dimensions is constructed using position vectors that locate the contact points. All applied forces or moments should be represented as vectors, and the application points of the forces should be specified with corresponding position vectors. The third step is to calculate the reaction forces at the six contact points by solving the simultaneous equations derived from static equilibrium. Neglecting friction, the directions of the reaction forces are known a priori since they are normal to the contacting surfaces. The sign of the reaction forces indicates whether the coupling remains in static equilibrium under the applied loads; if it is not, then both the geometry of the configuration and alternative configurations should be reconsidered.

If the coupling remains in static equilibrium for all possible assembly forces or moments, then the next step is to calculate the stress, strain, and elastic deformation produced at the contact points by the reaction forces. The final step is to determine the errors at the locations of the assembly operations that result from the translation or rotation of the pallet due to the elastic deformation at the contact points. This step is essential since small rotations of the pallet produce translation errors that are amplified by the distance to the assembly operation.

The geometry of a split-groove kinematic coupling is described with position vectors that are based in a Cartesian coordinate system located at the coupling centroid within the 


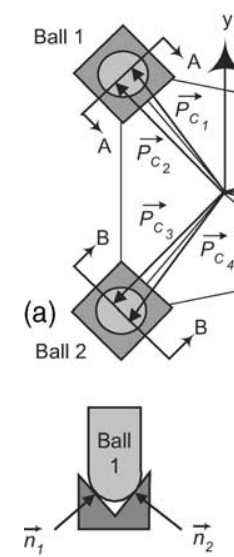

(b) Section A-A

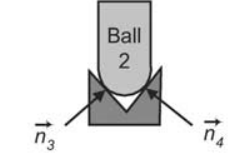

Section B-B

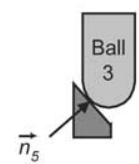

Section C-C
Fig. 10. Model of split-groove kinematic coupling using position and normal vectors.

plane of the balls' centers. Six position vectors, $\vec{P}_{\mathrm{C}_{i}}$, locate the contact points, and four position vectors, $\vec{P}_{\mathrm{B}_{j}}$, locate the balls' centers. Six unit vectors, $\vec{n}_{i}$, act through the contact points, normal to the contacting surfaces. The six normal vectors point in the direction from the groove surfaces and towards the balls' centers. These vectors are all illustrated in Fig. 10, and can be expressed parametrically as functions of the designer's preferred dimensions, e.g. coupling length, width, coupling angle, and ball diameter.

Following the approach presented by Schmiechen and Slocum [14], the equations of static equilibrium are written in matrix form as shown in Eq. (13). The system matrix, $[K]$, relates the applied loads, $\vec{L}_{\mathrm{A}}$, to the reaction forces at the contact points, $\vec{R}_{\mathrm{C}}$. $[K]$ is calculated using the position and normal vectors as shown in Eq. (14). $\vec{L}_{\mathrm{A}}$ is a $6 \times 1$ vector of forces and moments that is calculated as the sum of loads due

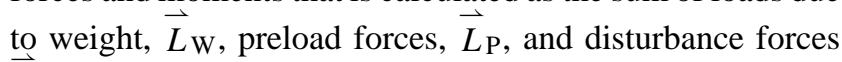
$\vec{L}_{\mathrm{D}}$ as shown in Eq. (15). Any forces or moments that arise due to assembly operations are treated as disturbance loads. The six reaction forces at the contact points are calculated as the components of the $6 \times 1$ vector $\vec{R}_{\mathrm{C}}$ :

$$
\begin{aligned}
& \vec{R}_{\mathrm{C}}=-[K]^{-1} \vec{L}_{\mathrm{A}} \\
& {[K]=\left[\begin{array}{cccc}
\vec{n}_{1} & \vec{n}_{2} & \vec{n}_{3} & \vec{n}_{4} \\
\vec{P}_{\mathrm{C}_{1}} \times \vec{n}_{1} & \vec{P}_{\mathrm{C}_{2}} \times \vec{n}_{2} & \vec{P}_{\mathrm{C}_{3}} \times \vec{n}_{3} & \vec{P}_{\mathrm{C}_{4}} \times \vec{n}_{4}
\end{array}\right.} \\
& \left(\vec{L}_{\mathrm{A}}\right)_{6 \times 1}=\vec{L}_{\mathrm{W}}+\vec{L}_{\mathrm{P}}+\vec{L}_{\mathrm{D}} \\
& =\left[\begin{array}{c}
\left(\vec{F}_{\mathrm{W}_{i}}\right)_{3 \times 1} \\
\left(\vec{P}_{\mathrm{W}} \times \vec{F}_{\mathrm{W}}\right)_{3 \times 1}
\end{array}\right]+\sum_{i=1}^{l}\left[\begin{array}{c}
\left(\vec{F}_{\mathrm{P}_{i}}\right)_{3 \times 1} \\
\left(\vec{P}_{\mathrm{P}_{i}} \times \vec{F}_{\mathrm{P}_{i}}\right)_{3 \times 1}
\end{array}\right] \\
& +\sum_{j=1}^{m}\left[\begin{array}{c}
\left(\vec{F}_{\mathrm{D}_{i}}\right)_{3 \times 1} \\
\left(\vec{P}_{\mathrm{D}_{i}} \times \vec{F}_{\mathrm{D}_{i}}\right)_{3 \times 1}
\end{array}\right]
\end{aligned}
$$

After determining the reaction forces in $\vec{R}_{\mathrm{C}}$, the distribution of stress near the contact points should be evaluated, and material failure should be considered. This is generally done assuming Hertzian contact $[19,20]$, and Slocum [21] provides a convenient form of the equations. The magnitude of the stress, strain, and deformation at the contact points depends upon the topography at the contact points (Fig. 9) and the contacting materials. Ductile materials are likely to fail due to shear stress beneath the contacting surface, and brittle materials are likely to fail due to tensile stress near the edge of the contact area. If a comparison of the maximum stresses with the material strength indicates failure, then the design can be improved by changing dimensions, materials, or contact topography.

In the three configurations of kinematic couplings illustrated in Fig. 7, a sphere contacts a flat surface. For this simple case, the contacting surfaces are axi-symmetric about the surface normal vector, and so the contact area at the $i$ th contact point is circular. The radius of the circular contact area, $a_{i}$, is given in Eq. (16) as a function of the reaction force at the contact point, $R_{\mathrm{C}_{i}}$, the diameter of the contacting sphere, $D_{\mathrm{S}_{i}}$, and the equivalent modulus of elasticity, $E_{\mathrm{e}}$. Eq. (17) gives $E_{\mathrm{e}}$ in terms of the moduli of elasticity and Poisson's ratios for the sphere and flat surfaces:

$$
\begin{aligned}
& a_{i}=\left(\frac{3 R_{\mathrm{C}_{i}} D_{\mathrm{s}_{i}}}{8 E_{\mathrm{e}}}\right)^{1 / 3}, \quad \text { for } i=1, \ldots, 6 \\
& E_{\mathrm{e}}=\frac{1}{\left(\left(1-v_{\mathrm{s}}^{2}\right) / E_{\mathrm{s}}\right)+\left(\left(1-v_{\mathrm{f}}^{2}\right) / E_{\mathrm{f}}\right)}
\end{aligned}
$$

For the case of a sphere contacting a flat surface, Johnson [20] gives simple expressions for the maximum shear stress and maximum tensile stress. Eq. (18) gives the maximum shear stress at the $i$ th contact point, $\tau_{\max }$, which should be considered for ductile material failure. The maximum tensile stress at the $i$ th contact point, $\sigma_{\max }$, should be considered for failure of brittle materials, and it depends upon Poisson's ratio for the contacting material and is given in Eq. (19):

$\tau_{\max _{i}} \approx \frac{0.47 R_{\mathrm{C}_{i}}}{\pi a_{i}^{2}}$

$$
\left.\begin{array}{cc}
\vec{n}_{5} & \vec{n}_{6} \\
\vec{P}_{\mathrm{C}_{5}} \times \vec{n}_{5} & \vec{P}_{C_{6}} \times \vec{n}_{6}
\end{array}\right]_{6 \times 6}
$$

$\sigma_{\max _{i}} \approx \frac{(1-2 v) R_{\mathrm{C}_{i}}}{2 \pi a_{i}^{2}}$

The compressive reaction forces at the contact points produce strain in the contacting bodies so that the $i$ th sphere and flat approach each other by distance, $\delta_{\mathrm{C}_{i}}$, which can be calculated with Eq. (20). The deformations at all six contact points are 
grouped into a $6 \times 1$ vector, $\vec{\delta}_{\mathrm{C}}$ :

$\delta_{\mathrm{C}_{i}}=\left(\frac{18 R_{\mathrm{C}_{i}}^{2}}{16 D_{\mathrm{S}_{i}} E_{\mathrm{e}}^{2}}\right)^{1 / 3}$

Due to the deformation at the six contact points, a rigid pallet translates and rotates with respect to a coordinate system in the machine base. Since this motion is undesirable, it is referred to as the pallet's error motion. The rigid body translations and rotations are components of a vector, $\vec{\delta}_{\mathrm{E}}$, as shown in Eq. (21), and they are measured in the Cartesian coordinates at the coupling's centroid (Fig. 10). As shown by Schmiechen and Slocum [14], $\vec{\delta}_{\mathrm{E}}$ can be calculated using the transpose of the inverted system matrix and the contact deformation vector as shown in Eq. (22):

$$
\begin{aligned}
& \vec{\delta}_{\mathrm{E}}=\left[\begin{array}{llllll}
\delta^{x} & \delta^{y} & \delta^{z} & \varepsilon^{x} & \varepsilon^{y} & \varepsilon^{z}
\end{array}\right]^{\mathrm{T}} \\
& \vec{\delta}_{\mathrm{E}}=\left([K]^{-1}\right)^{\mathrm{T}} \vec{\delta}_{\mathrm{C}}
\end{aligned}
$$

Homogenous transformation matrices (HTMs) provide an effective method to represent translations and rotations between successive coordinate systems, and an introduction to this approach is available from Craig [22]. The translations $\left(\delta^{x}, \delta^{y}, \delta^{z}\right)$ and rotations $\left(\varepsilon^{x}, \varepsilon^{y}, \varepsilon^{z}\right)$ associated with the pallet's error motion are therefore collected in the HTM given in Eq. (23), assuming small rotation angles. $\left[T_{\mathrm{E}}\right]$ represents the transformation between coordinate systems at the coupling centroid in the machine base and pallet that differ by the error motion. The position of a point on the pallet or fixture after the error motion is then determined by premultiplying a position vector that locates the point with $\left[T_{\mathrm{E}}\right]$ :

$$
\left[T_{\mathrm{E}}\right]=\left[\begin{array}{cccc}
1 & -\varepsilon^{z} & \varepsilon^{y} & \delta^{x} \\
\varepsilon^{z} & 1 & -\varepsilon^{x} & \delta^{y} \\
-\varepsilon^{y} & \varepsilon^{x} & 1 & \delta^{z} \\
0 & 0 & 0 & 1
\end{array}\right]
$$

Small rotations that occur at the coupling centroid produce displacement errors at other positions on the pallet or fixture. To assess this effect on assembly operations, it is necessary to calculate the position error, $\vec{P}_{\mathrm{E}}$, at each point on the fixture or pallet where assembly operations are conducted. Each operating point should be located with a position vector, $\vec{P}_{\mathrm{o}}$, measured in the coupling's centroid coordinate system. The position error, expressed in Eq. (24), is then the difference between the position of the operating point before and after the error motion:

$\vec{P}_{\mathrm{E}}=\left[T_{\mathrm{E}}\right] \vec{P}_{\mathrm{o}}-\vec{P}_{\mathrm{o}}$

\subsection{Conditions for tipping due to disturbance loads}

It is likely that assembly operations will subject a pallet to a range of disturbance forces, and so the combination of the pallet weight and preload forces should be sufficient to pre- vent tipping or sliding of a kinematically coupled pallet. This condition is satisfied when contact exists at the six contact points as indicated when the reaction forces at the contact points are all positive. This condition is expressed in Eq. (25), where $R_{\mathrm{C}_{i}}$ is the reaction at the $i$ th contact point and a component of the reaction force vector, $\vec{R}_{\mathrm{C}}$ :

$R_{\mathrm{C}_{i}}>0, \quad$ for $i=1, \ldots, 6$

The condition may be rewritten in terms of the applied loads and row vectors from the inverted system matrix [2]. In Eq. (26), the condition is expressed as the dot product between the $i$ th row vector of the inverted system matrix, $\vec{K}_{R_{i}}^{-1}$, and the disturbance load, $\vec{L}_{\mathrm{D}}$, should be less than the dot product between $\vec{K}_{R_{i}}^{-1}$ and the sum of the loads due to weight and preload. As demonstrated in the following section, this condition can be used to graphically visualize a bounded region, limited by the six values on the right hand side of Eq. (26), in which the kinematic coupling should not tip due to in sufficient preloading:

$$
\begin{aligned}
& \left(\vec{K}_{R_{i}}^{-1}\right)_{6 \times 1}\left(\vec{L}_{\mathrm{D}}\right)_{6 \times 1} \\
& <\left(\vec{K}_{R_{i}}^{-1}\right)_{6 \times 1}\left(\left[\begin{array}{c}
\left(\vec{F}_{\mathrm{W}}\right)_{3 \times 1} \\
\left(\vec{P}_{\mathrm{W}} \times \vec{F}_{\mathrm{W}}\right)_{3 \times 1}
\end{array}\right]\right. \\
& \left.\quad+\sum_{i=1}^{l}\left[\begin{array}{c}
\left(\vec{F}_{\mathrm{P}_{i}}\right)_{3 \times 1} \\
\left(\vec{P}_{\mathrm{P}_{i}} \times \vec{F}_{\mathrm{P}_{i}}\right)_{3 \times 1}
\end{array}\right]\right), \text { for } i=1, \ldots, 6
\end{aligned}
$$

\subsection{Pallets employing split-groove kinematic couplings}

The procedures described in Sections 3.1 through 3.3 were applied in the design of a split-groove kinematic coupling for improving the commercial pallet system [2]. The pins and bushings within the commercial pallet were not used; instead, a split-groove kinematic coupling was incorporated into a fixture that fastened onto the commercial pallet. Hard stainless steel (440C) tooling balls were fastened to the fixture plate, and hard stainless steel (440C) vee-grooves were incorporated into the assembly workstation. The split-groove configuration made it possible for the vee-grooves to straddle the width of the pallets' conveyor system and eliminated the need for a vee-groove in the center of the conveyor system. The pallet was preloaded into the vee-grooves using a set of 12 permanent magnets, six in the fixture plate and six in the machine base. The magnetic preload was sufficient to ensure that the pallet remained in equilibrium for a range of disturbance forces, but the preload was not excessive for manually removing the pallet off of the workstation. Fig. 11 shows photographs of the split-groove kinematic pallet system. The vee-grooves and the permanent magnets in the workstation base can be seen in Fig. 11a. Fig. 11b shows the bottom of the pallet plate that contains four balls and six preload magnets. Fig. 11c shows the fixture and pallet kinematically coupled to the workstation base. 


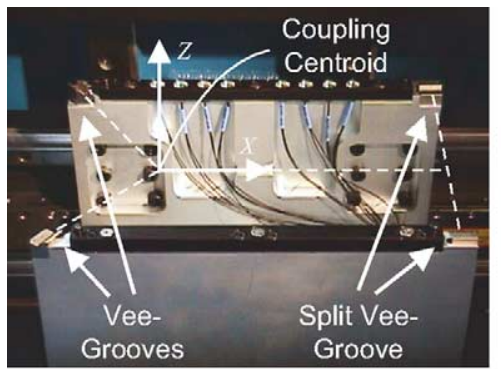

(a)

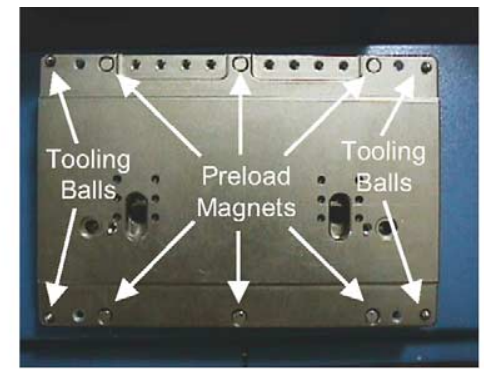

(b)

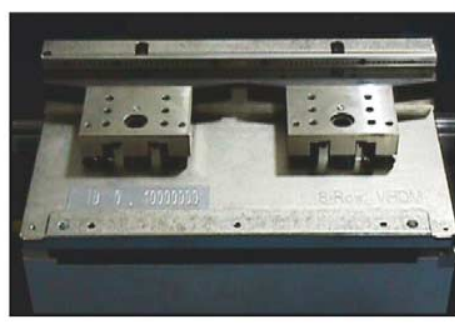

(c)

Fig. 11. Split-groove kinematically coupled pallet and assembly workstation. (a) Machine base with vee-grooves and preload magnets. (b) Bottom of fixture plate with tooling balls and preload magnets. (c) Pallet and fixture plate kinematically coupled to machine.

The dimensions of the split-groove kinematic coupling are listed in Table 2. Using these dimensions, the position vectors to the contact points and normal vectors illustrated in uated using Eqs. (20)-(24). These calculations are not presented here, since examples are available in prior literature by Schmiechen and Slocum [14] and Johnson [20]:

$$
[K]=\left[\begin{array}{cccccc}
-0.578 & 0.578 & 0.578 & -0.578 & 0.000 & 0.000 \\
-0.408 & 0.408 & -0.408 & 0.408 & 0.707 & -0.707 \\
0.707 & 0.707 & 0.707 & 0.707 & 0.707 & 0.707 \\
71.842 & 71.842 & -71.842 & -71.842 & -69.597 & 69.597 \\
50.773 & 50.773 & 50.773 & 50.773 & -152.103 & -152.103 \\
87.973 & -87.973 & 87.973 & -87.973 & 152.103 & -152.103
\end{array}\right]
$$

Fig. 10 are determined, and the components of the vectors are summarized in Tables 3 and 4 . The system matrix, $[K]$, shown in Eq. (27) is calculated by substituting the components of the position vectors and normal vectors into Eq. (14). Knowing the system matrix and any applied forces enables the determination of the reaction forces at the contact points with Eq. (13). The critical contact stress at each contact point is determined using the Hertzian relations for a sphere contacting a flat surface, Eqs. (16)-(19), and the errors are eval-

Table 2

Dimensions of split-groove kinematic coupling

\begin{tabular}{lc}
\hline Parameter & Dimension \\
\hline Coupling length $(\mathrm{mm})$ & 286.91 \\
Coupling width $(\mathrm{mm})$ & 203.20 \\
Split offset (mm) & 196.85 \\
Groove angle (radian) & $\pi / 2$ \\
Ball diameter (mm) & 6.35 \\
\hline
\end{tabular}

Table 3

Components of position vectors that locate the six contact points in coordinate system at coupling centroid

\begin{tabular}{lcrc}
\hline Description & \multicolumn{3}{l}{ Vector } \\
\cline { 2 - 4 } & $X(\mathrm{~mm})$ & $Y(\mathrm{~mm})$ & $Z(\mathrm{~mm})$ \\
\hline Contact Point 1 & -69.97 & 102.90 & -2.25 \\
Contact Point 2 & -73.64 & 100.30 & -2.25 \\
Contact Point 3 & -73.64 & -100.30 & -2.25 \\
Contact Point 4 & -69.97 & -102.90 & -2.25 \\
Contact Point 5 & 215.11 & -100.67 & -2.25 \\
Contact Point 6 & 215.11 & 100.67 & -2.25 \\
\hline
\end{tabular}

Since the pallet is subject to a range of disturbance forces, it is necessary to ensure that the pallet will not tip using the condition presented in Section 3.3. Within the assembly workstation, the forces applied to the kinematically coupled pallet include the pallet's weight, six preload forces, and disturbance forces that occur during the assembly operations. The weight of the pallet is approximately $64.7 \mathrm{~N}$, and the preload is the result of six pairs of neodymium permanent magnets that each generates a force of about $22.3 \mathrm{~N}$. During assembly operations, an insertion force of about $135 \mathrm{~N}$ presses downward on the pallet. The insertion forces are applied along a line that is parallel to the $x$-axis between $x=-96.84$ and $225.3 \mathrm{~mm}$; the $y$ - and $z$-coordinate are -44.37 and $89.10 \mathrm{~mm}$, respectively. A summary of the force components and the position vectors that locate the application points of the forces are listed in Table 5.

The six conditional functions given in Eq. AAA) were determined from Eq. (26) and are associated with each contact point. Evaluating the right-side of Eq. (26) yields a constant value, but the left-side of the inequality is a function of the

Table 4

Components of normal vectors at the six contact points

\begin{tabular}{lrrr}
\hline Description & \multicolumn{1}{l}{ Vector } & \\
\cline { 2 - 4 } & $X(\mathrm{~mm})$ & $Y(\mathrm{~mm})$ & $Z(\mathrm{~mm})$ \\
\hline Normal Vector 1 & -0.5775 & -0.4081 & 0.7071 \\
Normal Vector 2 & 0.5775 & 0.4081 & 0.7071 \\
Normal Vector 3 & 0.5775 & -0.4081 & 0.7071 \\
Normal Vector 4 & -0.5775 & 0.4081 & 0.7071 \\
Normal Vector 5 & 0.0000 & 0.7071 & 0.7071 \\
Normal Vector 6 & 0.0000 & -0.7071 & 0.7071 \\
\hline
\end{tabular}


Table 5

Forces applied to split-groove kinematic pallet

\begin{tabular}{|c|c|c|c|c|c|c|}
\hline \multirow[t]{2}{*}{ Force description } & \multicolumn{3}{|c|}{ Force components } & \multicolumn{3}{|l|}{ Position vector } \\
\hline & $X(\mathrm{~N})$ & $Y(\mathrm{~N})$ & $Z(\mathrm{~N})$ & $X(\mathrm{~mm})$ & $Y(\mathrm{~mm})$ & $Z(\mathrm{~mm})$ \\
\hline Weight & 0.00 & 0.00 & -64.7 & 71.98 & -8.80 & 7.07 \\
\hline Preload 1 & 0.00 & 0.00 & -22.3 & -28.73 & 99.31 & 1.78 \\
\hline Preload 2 & 0.00 & 0.00 & -22.3 & 72.87 & 99.31 & 1.78 \\
\hline Preload 3 & 0.00 & 0.00 & -22.3 & 174.5 & 99.31 & 1.78 \\
\hline Preload 4 & 0.00 & 0.00 & -22.3 & -28.73 & -99.31 & 1.78 \\
\hline Preload 5 & 0.00 & 0.00 & -22.3 & 72.87 & -99.31 & 1.78 \\
\hline Preload 6 & 0.00 & 0.00 & -22.3 & 174.5 & -99.31 & 1.78 \\
\hline Assembly forces & 0.00 & 0.00 & -135 & -96.84 to 225.3 & -44.37 & 89.10 \\
\hline
\end{tabular}

component of the insertion force in the $z$ direction, $F_{\mathrm{D}}^{z}$, which may vary between 0 and $-135 \mathrm{~N}$, and the $x$-coordinate where the insertion force is applied, $P_{\mathrm{D}}^{x}$, which may vary between -96.84 and $225.3 \mathrm{~mm}$. In this particular case, the conditional functions for contact Points 1 and 2 are identical, the functions for Points 3 and 4 are identical, and the functions for Points 5 and 6 are identical:
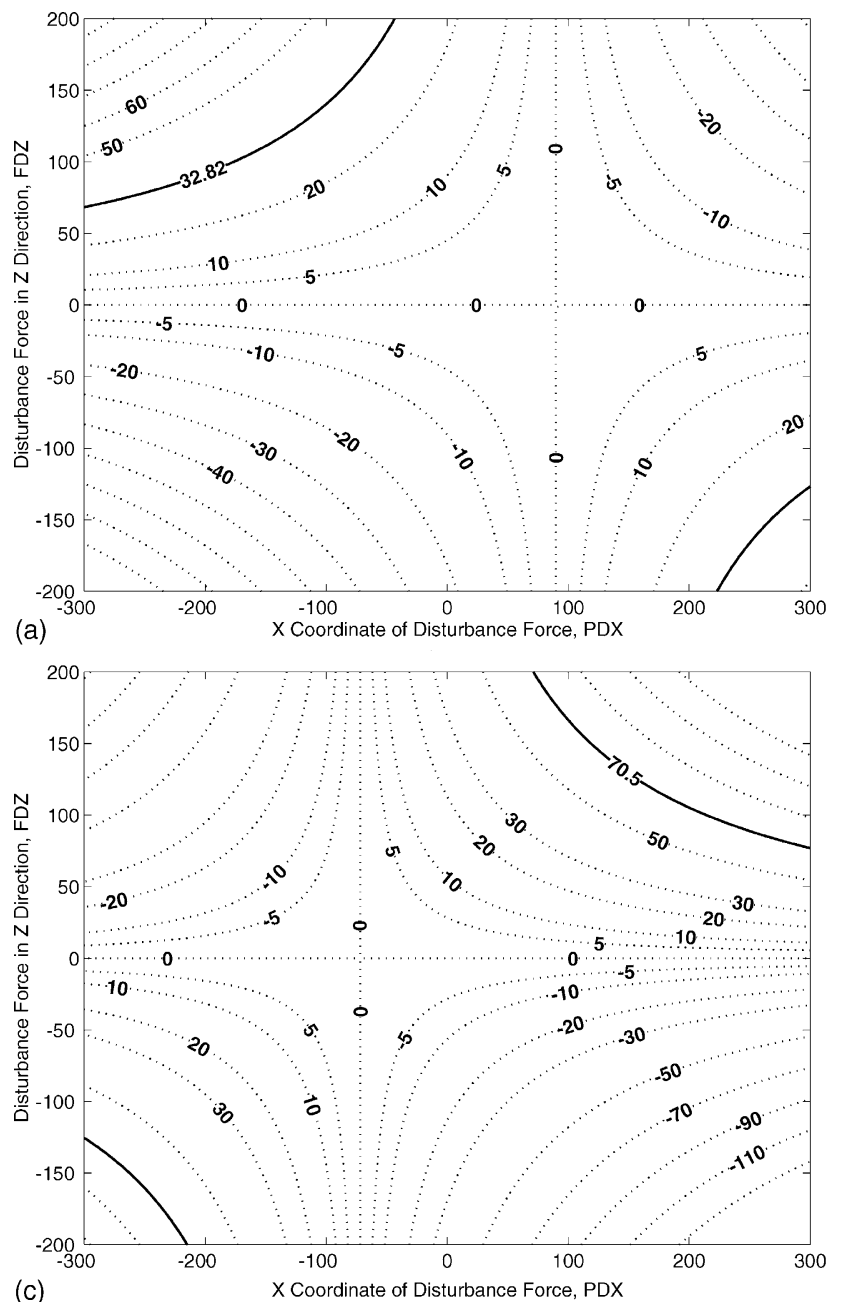

$$
\begin{aligned}
& 0.265 F_{\mathrm{D}}^{z}-44.37(0.0035) F_{\mathrm{D}}^{z}-0.0012\left(P_{\mathrm{D}}^{x} F_{\mathrm{D}}^{z}\right)<32.8 \\
& 0.265 F_{\mathrm{D}}^{z}-44.37(0.0035) F_{\mathrm{D}}^{z}-0.0012\left(P_{\mathrm{D}}^{x} F_{\mathrm{D}}^{z}\right)<32.8 \\
& 0.265 F_{\mathrm{D}}^{z}+44.37(0.0035) F_{\mathrm{D}}^{z}-0.0012\left(P_{\mathrm{D}}^{x} F_{\mathrm{D}}^{z}\right)<36.8 \\
& 0.265 F_{\mathrm{D}}^{z}+44.37(0.0035) F_{\mathrm{D}}^{z}-0.0012\left(P_{\mathrm{D}}^{x} F_{\mathrm{D}}^{z}\right)<36.8 \\
& 0.177 F_{\mathrm{D}}^{z}+0.0025\left(P_{\mathrm{D}}^{x} F_{\mathrm{D}}^{z}\right)<70.5
\end{aligned}
$$
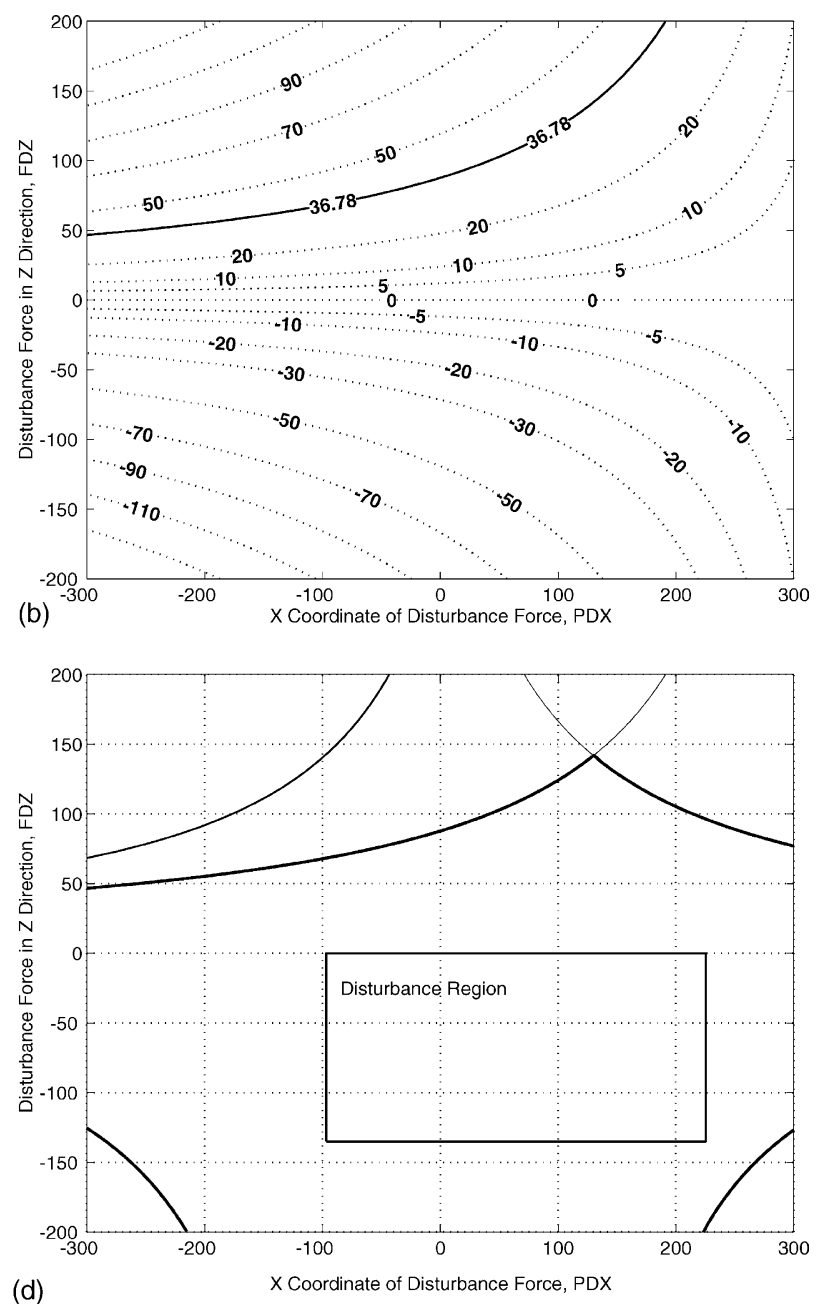

Fig. 12. Contour plots of the conditions for equilibrium at (a) contact Points 1 and 2, (b) contact Points 3 and 4, (c) contact Points 5 and 6, and (d) intersection of the conditions and the disturbance region. 


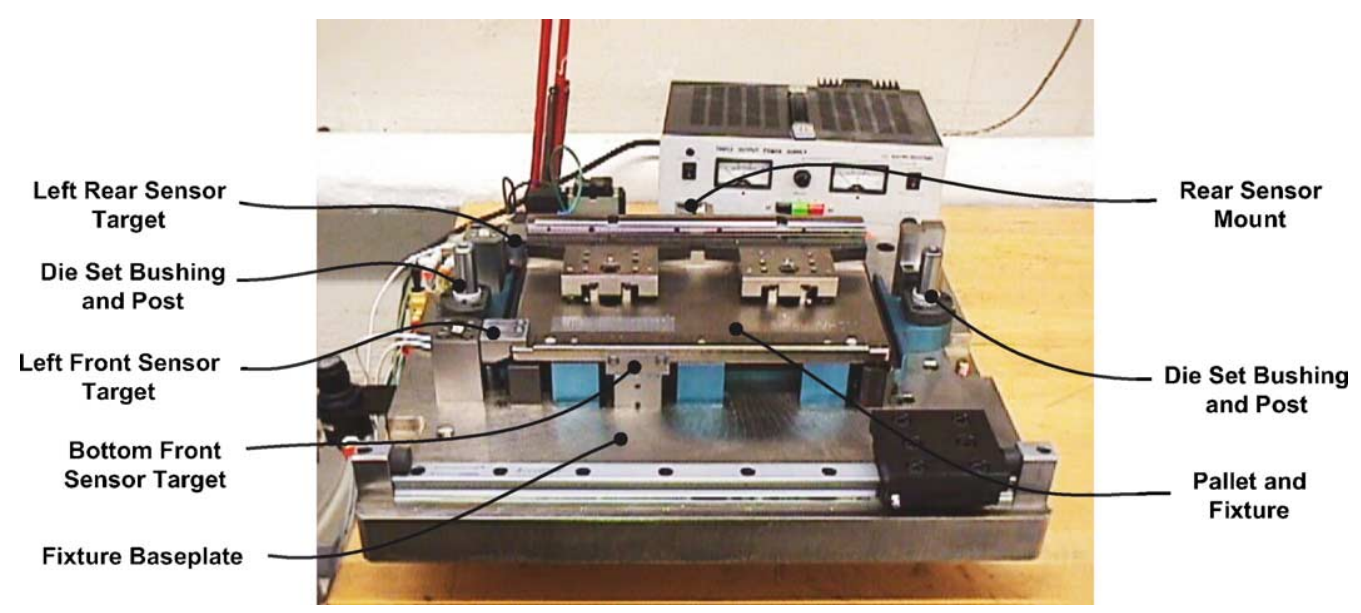

Fig. 13. Experimental setup for measuring pallet repeatability.

The values on the left side of the inequalities are plotted as contours in Fig. 12a-c. A bold contour indicates the boundary associated with the constants given on the right side of Eq. (28). In the regions where values in the contour plots are less than the constant, the corresponding ball remains in contact with the flat surface. Fig. 12d shows the intersection of the boundaries for each contact point and the boundaries of the disturbance region. Since the equilibrium boundaries are outside the disturbance region, the pallet remains in equilibrium without tipping.

\subsection{Precision of kinematically coupled pallets}

Kinematic couplings are known for their ability to precisely position one object with respect to another with extreme repeatability $[16,25]$. The principal reason for any non-repeatable behavior is the interaction between friction and structural compliance. Although analytical methods for relating design parameters to repeatability are not thoroughly developed, Hale suggested the relation given in Eq. (29) as an estimate of repeatability [23]. Hale derived the estimate as if the coupling's compliance in all directions is equal to a single Hertzian contact carrying a load $P$ and having a relative radius, $R$, and elastic modulus, $E$ [24]:

$\rho \equiv \frac{f}{k} \approx \mu\left[\frac{2}{3 R}\right]^{1 / 3}\left[\frac{P}{E}\right]^{2 / 3}$

Since friction forces are uncertain and inconsistent, analytical techniques for assessing repeatability should be treated only as estimates. For this reason, accurate assessments of a kinematic coupling's repeatability should be determined experimentally. For instance, Slocum experimentally measured the repeatability of a three-groove coupling (356 $\mathrm{mm}$ diameter) and found that its axial and radial repeatability were on the order of $0.30 \mu \mathrm{m}$ [25]. Schouten and Schellekens measured the repeatability of a kinematic coupling that incorporated flexures for tangential compliance at the contact points and found that the hys- teresis was reduced by a factor of $10 \times$ to less than $0.1 \mu \mathrm{m}$ [16].

The repeatability of the split-groove kinematic pallet shown in Fig. 11 was assessed experimentally. The experiments were conducted on the apparatus shown in Fig. 13, which duplicated the geometry of the machine base in the assembly workstation, including the vee-grooves, the split vee-groove, and the preload magnets. The pallet was lifted and dropped onto the grooves by a pneumatically actuated lift-plate. The lift-plate was guided by die set bushings, and it separated from the pallet at the bottom of travel to prevent over constraining the pallet's position. Capacitive displacement sensors were mounted around the perimeter of the pallet to measure the variation in the position of the pallet. Each sensor provided an analog signal $( \pm 10 \mathrm{~V})$ proportional to the change in the distance between the face of the sensor and a steel target attached to the pallet $( \pm 50 \mu \mathrm{m})$. A 16-bit data acquisition system measured signals between $\pm 5 \mathrm{~V}$, providing a resolution around $0.76 \mathrm{~nm} / \mathrm{bit}$.

In factory-like environmental conditions, the split-groove kinematic pallet was lifted and dropped thousands of times over 2 days, and the gap distance was measured each time. Fig. 14 shows a plot of the four sensors' displacement measurements in microns. The relative expansion and contraction of the pallet and fixture due to ambient temperature

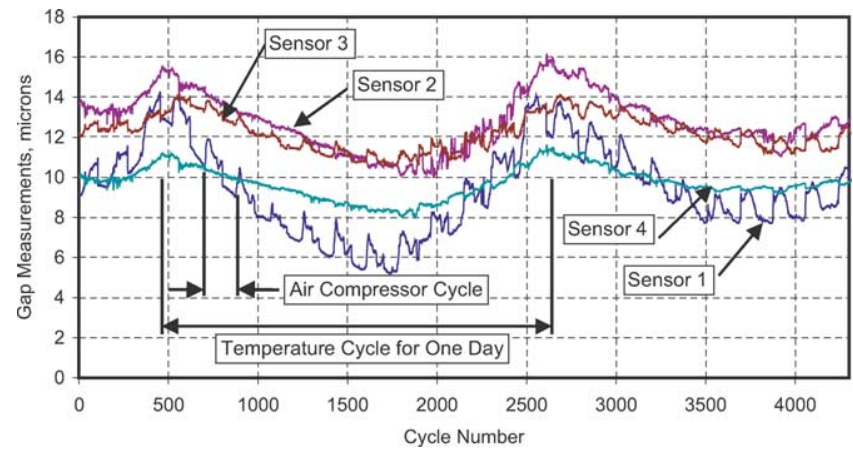

Fig. 14. Repeatability measurements for split-groove kinematic pallet. 
cycles produced cyclic fluctuations with a period of about $24 \mathrm{~h}$ and maximum amplitude of about $10 \mu \mathrm{m}$ in Sensor 1. The pneumatic system's air pressure produced additional periodic fluctuations with a period of approximately $2 \mathrm{~h}$. Even with the periodic fluctuations associated with the thermal and pneumatic cycles, the pallet was repeatable to within $\pm 5 \mu \mathrm{m}$. This represents a $10 \times$ improvement over the conventional pallet that employed pins-in-holes, which is only repeatable to about $\pm 50 \mu \mathrm{m}$.

\section{Conclusion}

Mass customization strategies are generating renewed interested in flexible manufacturing and assembly systems, even in products requiring high precision. To reduce the amount of manufacturing variation, it is necessary to consider the precision of alternative techniques for locating pallets at workstations. The conventional approach uses pins-in-holes, but the precision of this technique is often limited by the clearances between locating bushings and pins. A method for estimating the amount of expected variation in the position and orientation of a pallet that uses the pins-in-holes technique was presented. Kinematic couplings provide an alternative technique, which exactly constrains the location and orientation of the pallet. The procedure and analyses used in designing kinematically couplings is presented and demonstrated in designing a split-groove kinematic coupling for assembly pallets, which gives the appearance of four-point support thus enhancing pallet stability. Experimental measurements of the kinematically coupled pallet demonstrated an order of magnitude reduction in the variability of the pallet's location compared to the conventional pins-in-holes technique. Therefore, split-groove kinematic couplings provide great advantage for achieving precise locations of pallets in multi-station assembly systems. With appropriate consideration of preload forces, the split-groove kinematic coupling can be effective when conventional three-groove couplings are not feasible due to geometric constraints.

\section{Acknowledgments}

This work was supported by Teradyne Connection Systems and a grant from the Defense Logistics Agency AQP.

\section{References}

[1] Bosch catalog for TS-2 conveyor system. Bosch Automation Products. 816 E. Third St., Buchanan, MI 49107. Publication No. 8981500127.
[2] Vallance RR. Precision connector assembly automation. Ph.D. Dissertation, Massachusetts Institute of Technology; 1999.

[3] Blanding DL. Exact constraint: machine design using kinematic principles. New York: ASME Press; 1999.

[4] Evans C. Precision engineering: an evolutionary view. Bedford, UK: Cranfield Press; 1989.

[5] Jones RV. Anti-distortion mountings for instruments and apparatus. J Sci Instrum 1961;38:408-9.

[6] Whipple RS. The design and construction of scientific instruments. Trans Opt Soc 1920/1921;22:3-52.

[7] Brooks LS. Adjustable instrument mount. J Opt Soc Am 1954;44: $107-9$.

[8] Hog E. A kinematic mounting. Astronomics Astrophys 1975;41: $107-9$.

[9] Battles AE, Linder BM, Chang KW, Slocum AH. The design of a precision Bilaminar resonating transducer assembly tool. Precision Eng 1993, October;15(4):248-57.

[10] Culpepper ML, Slocum AH. Quasi-kinematic couplings: a low-cost method for precision coupling of product components and the like in manufacturing processes. In: Proceedings of the 1999 Annual Meeting of the American Society for Precision Engineering, Monteray, CA; November 1999.

[11] Hale LC. Principles and techniques for designing precision machines. Ph.D. Thesis, Massachusetts Institute of Technology; February 1999.

[12] Donaldson R. The deterministic approach to machining accuracy. In: Proceedings of the SME Fabrication Technolology Symposium, Golden, CO; November 1972 (UCRL Preprint 74243).

[13] Slocum AH. Design of three-groove kinematic couplings. Precision Eng 1992;14(2):67-76.

[14] Schmiechen P, Slocum AH. Analysis of kinematic systems: a generalized approach. Precision Eng 1996, July;19(1):11-8.

[15] Van Doren MJ. Precision machine design for the semiconductor equipment manufacturing industry. Doctoral Thesis, Massachusetts Institute of Technology, Cambridge, MA, USA; May 1995.

[16] Schouten R, Schellekens. Design of a kinematic coupling for precision applications. Precision Eng 1997;20:46-52.

[17] Slocum AH. Design of new precision machine elements. Initiatives of precision engineering at the beginning of a millennium. In: Proceedings of the 10th International Conference on Precision Engineering (ICPE); 18-20 July 2001. p. 18-24.

[18] Olasz JS, MacIndoe WC. Surface finish, displacement, and contour scanner. U.S. Patent and Trademark Office. Patent \#4,574,625; 11 March 1986.

[19] Hertz H. On the contact of elastic solids. In: Jones, Schott, editors. Miscellaneous papers by H. Hertz. London: Macmillan; 1896.

[20] Johnson KL. Contact mechanics. Cambridge, UK: Cambridge University Press; 1985.

[21] Slocum AH. Precision machine design. Society of Manufacturing Engineers; 1992.

[22] Craig JJ. Introduction to robotics, mechanics and control. New York: Addison-Wesley; 1989.

[23] Hale LC. Friction-based design of a kinematic coupling for precision applications. In: Proceedings of the American Society for Precision Engineering Annual Conference; 1998. p. 45-8.

[24] Hale LC, Slocum AH. Optimal design techniques for kinematic couplings. Precision Eng 2001;25(2):114-27.

[25] Slocum A. Kinematic couplings for precision fixturing-Part 1: Formulation of design parameters. Precision Eng 1988, April;10(2): $85-91$. 